\title{
Value-based healthcare: II nuovo approccio di AIFA alla determinazione multidimensionale del valore
}

\author{
Oriana Ciani $^{1,2}$, Carlo Federici ${ }^{1}$, Giulia Fornaro ${ }^{1}$, Carla Rognoni ${ }^{1}$ \\ ${ }^{1}$ CERGAS, SDA Bocconi, Milano - Italy \\ ${ }^{2}$ College of Medicine \& Health, University of Exeter, Exeter - UK
}

\begin{abstract}
AIFA's new approach to multidimensional value determination
In this viewpoint, we discuss the approach promoted by the Italian Drug Agency (AIFA) to the assessment of the value of new pharmaceuticals in the Italian Healthcare Service. On top of traditionally acknowledged components, such as quality adjusted life years gained and net costs, the overall value framework might include other elements such as productivity and adherence, equity, severity of disease, reduction in uncertainty, spillover effects. There is a residual dimension in the value framework that may capture the option value or reduction in fear of contagion for infectious disease treatments. We debate measurement issues on these elements of value and discuss open issues from a methodological and policy standpoint.
\end{abstract}

Keywords: AIFA, HTA, Value, Value-based healthcare (VBHC)

\section{Introduzione}

Negli ultimi anni il passo dell'innovazione tecnologica da un lato, e la pressione per il contenimento dei costi a fronte di budget limitati dall'altro, hanno promosso a livello internazionale l'affermazione del paradigma che va sotto il nome di Health Technology Assessment (HTA), ovvero un approccio sistematico, multidimensionale e multidisciplinare a supporto delle decisioni di finanziamento o rimborsabilità delle tecnologie sanitarie (1).

La valutazione dell'impatto economico e finanziario costituisce una componente essenziale del processo di HTA. La letteratura di riferimento (2) definisce le valutazioni economiche come analisi volte a misurare e valutare i costi (valutazioni economiche parziali-analisi dei costi, analisi di impatto sul budget) insieme agli esiti di salute di due o più alternative terapeutiche (valutazioni economiche complete-analisi costo-beneficio, costo-efficacia, costo-utilità e di minimizzazione dei costi).

A livello nazionale, il concetto di costo-efficacia e di sostenibilità rispetto alla negoziazione del prezzo e della rimborsabilità dei medicinali a carico del Servizio Sanitario Nazionale

Received: February 17, 2020

Accepted: April 8, 2020

Published online: May 6, 2020

Corresponding author:

Oriana Ciani, PhD

Via Rontgen 1

20136 Milano, Italia

oriana.ciani@unibocconi.it
(SSN) è stato introdotto per la prima volta nel 1997 (Delibera CIPE n. 5, 30 gennaio 1997), poi successivamente richiamato dalla Delibera CIPE n. 3, del $1^{\circ}$ febbraio 2001, e definitivamente adottato con delibera 8 aprile 2016 n. 8, che integra concretamente le valutazioni economiche nelle procedure di valutazione da parte della Commissione Tecnico-Scientifica (CTS) e del Comitato Prezzi e Rimborso (CPR) dell'Agenzia Italiana del Farmaco (AIFA).

La contrattazione tra AIFA e le aziende farmaceutiche (Legge del 24 novembre 2003, n.326) per la determinazione del prezzo del medicinale si basa infatti su un dossier che le aziende farmaceutiche devono sottoporre all'AIFA, nell'ambito del quale l'azienda è invitata a supportare la proposta di prezzo e di rimborsabilità sulla base di valutazioni farmacoeconomiche a cui è riservata una sezione dedicata del rapporto (3). Per la valutazione critica di questo capitolo, AIFA adotta standard riconosciuti a livello internazionale $(2,4,5)$. Rispetto alla misurazione dell'esito di salute, un tema su cui si dibatte da tempo con posizioni divergenti da parte di Agenzie di HTA diverse, AIFA appoggia l'uso degli anni di vita pesati per la qualità (Quality-Adjusted Life Years, QALY). In estrema sintesi, questo indicatore di esito esprime il beneficio relativo a una tecnologia sanitaria in termini sia di quantità, sia di qualità della vita guadagnata, quest'ultima spesso misurata attraverso strumenti validati come il questionario EuroQol 5D (EQ-5D) o il Short Form 6D (SF-6D) (6). Seppur con la consapevolezza dei limiti di questa metrica nel cogliere tutti gli elementi che concorrono alla determinazione del valore (7), I'uso dei QALY permette di confrontare tecnologie sanitarie anche molto diverse tra loro per tipologia o area terapeutica (ad esempio cardiovascolare e oncologica). La necessità di operare tali confronti, che sottende l'utilizzo delle valutazioni economiche in sanità, risponde infatti all'esigenza di definire 
in maniera strutturata e sistematica quale sia il valore relativo di una tecnologia, e se l'acquisizione e implementazione della stessa nel SSN rappresentino un uso efficiente delle limitate risorse disponibili (8).

Tuttavia, negli ultimi anni si è assistito a un rinnovato dibattito sulla definizione di valore relativamente alle tecnologie sanitarie, inclusi i farmaci, i dispositivi medici e tutti gli interventi sanitari in generale (9). Nel 2019 l'International Society of Pharmacoeconomics and Outcomes Research (ISPOR) ha incluso tra i top trend (10) in materia di farmacoeconomia la definizione di value assessment frameworks, ovvero sistemi multidimensionali di definizione del valore da applicare nel contesto della valutazione di una tecnologia sanitaria e che riflettono prospettive di stakeholders diversi (ad esempio quelle del paziente, dell'azienda sanitaria e della società nel complesso). Alla base dell'uso dei value frameworks risiede la convinzione che, se l'analisi di costo-efficacia è un utile punto di partenza per informare le decisioni del decisore e del pagatore, ulteriori elementi normalmente non inclusi in una valutazione economica "standard" possono risultare rilevanti per alcuni livelli decisionali.

Pochi mesi fa è comparso sul sito della nostra agenzia del farmaco - https://www.aifa.gov.it/valutazioni-economiche il grafico riportato nella Figura 1. Questa immagine illustra quali siano i costrutti potenzialmente rilevanti nella definizione del valore di un medicinale rimborsato non solo nella prospettiva del SSN, ma più in generale per la società. Non è detto che queste siano necessariamente tutte le dimensioni che AIFA intenda fattivamente considerare. In ogni caso, gli studiosi e la ricerca dovranno dimostrare con quali metodologie e con quale impatto integrare nei processi decisionali dimensioni di valore ad oggi non valutate o non valutabili.

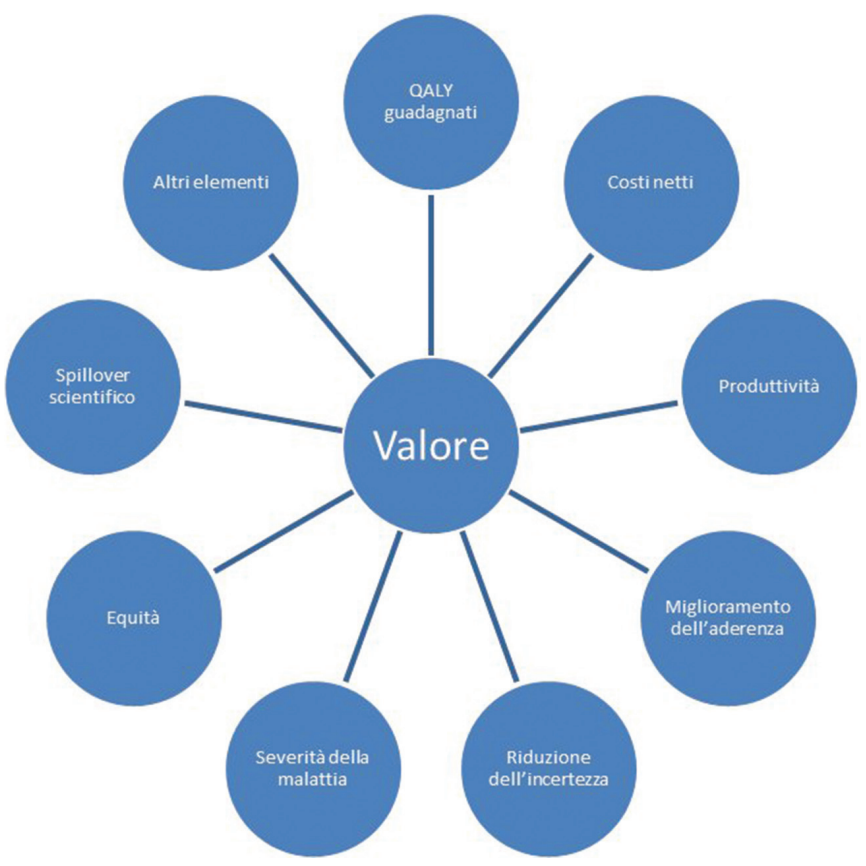

Fig. 1 - Dimensioni del valore secondo AIFA

Fonte: https://www.aifa.gov.it/valutazioni-economiche

\section{QALY guadagnati e costi netti}

Nel dibattito scientifico e professionale rispetto a ciò che merita considerazione nella determinazione del valore di una tecnologia sanitaria, vi è una convergenza su alcuni elementi, come la misurazione del beneficio clinico e del consumo di risorse relativi all'introduzione della tecnologia nel sistema sanitario. In particolare, il valore è associato a un profilo accettabile del confronto tra il miglioramento atteso degli esiti di salute e la differenza (spesso un incremento) dei costi rispetto a una o più alternative rilevanti. La relazione tra il beneficio ottenuto e il consumo di risorse necessario a ottenerlo viene solitamente espressa attraverso il rapporto incrementale di costo-efficacia (Incremental Cost-Effectiveness Ratio, ICER), che stima il costo incrementale che il sistema sanitario dovrebbe sostenere per ciascun paziente per ottenere un anno aggiuntivo di vita in piena salute. Nella rosa delle dimensioni del valore di AIFA, costi netti e QALY guadagnati riflettono questi due elementi del valore, ribadendo il supporto di AIFA all'utilizzo dei QALY come metrica di misurazione del beneficio clinico che combina sopravvivenza e qualità di vita (11).

\section{Produttività}

La quantificazione monetaria delle perdite o degli incrementi di produttività nell'ambito del lavoro è una dimensione teoricamente ben studiata, ma di fatto poco considerata nelle valutazioni economiche, che generalmente assumono la prospettiva del pagatore e non del datore di lavoro o della società nel complesso. Di fatto, la scarsa diffusione delle analisi di costo-beneficio, che utilizzano la più ampia prospettiva della società, è dovuta a storiche resistenze legate alla necessità di monetizzare le perdite di produttività in queste analisi, magari in funzione del reddito degli individui. Per evitare di introdurre disuguaglianze tra individui in età lavorativa e non, e classi socio-economiche diverse, la misurazione dovrebbe riguardare il tempo "produttivo" valorizzato in base a un salario medio generico più benefit adeguati al contesto di riferimento.

Una metodologia suggerita per incorporare i più ampi effetti a livello della società, all'interno di una analisi economica, riguarda ad esempio l'uso delle analisi di costo-conseguenza, dove i diversi effetti relativi all'introduzione di una tecnologia, come quelli sulla salute e sulla perdita di produttività dei pazienti e dei loro caregivers, vengono riportati separatamente nell'analisi, lasciando poi ai decisori il compito di valutare e prioritizzare le diverse dimensioni secondo un processo informale e soggettivo. Questa metodologia permette di rappresentare le conseguenze ampie dell'introduzione di una tecnologia sanitaria, ovviando però alla necessità di attribuire un valore monetario a tutte le dimensioni identificate (12).

Gli approcci alla valorizzazione delle perdite di produttività sono diversi, ognuno presenta vantaggi e svantaggi e andrebbe stabilito quale sia la strategia preferibile per la negoziazione sul prezzo e rimborso in AIFA.

\section{Miglioramento dell’aderenza}

Migliorare l'aderenza, per esempio attraverso schedule o modalità di somministrazione diverse, può avere effetti sia in 
termini di esiti clinici (migliorare l'aderenza dovrebbe significare migliorare l'efficacia clinica della terapia), sia in termini di costi (aumentando i costi diretti dell'uso del farmaco ma indirettamente riducendo il consumo di risorse dovuto a migliori esiti), e quindi incidere sul valore reale del farmaco. Pertanto, più che una dimensione di valore a sé stante, gli effetti del miglioramento dell'aderenza, e le eventuali differenze tra le tecnologie valutate, dovrebbero essere opportunamente quantificati e incorporati all'interno delle analisi di costo-efficacia (CEA). L'impatto del miglioramento dell'aderenza rispetto al profilo di costo-efficacia potrebbe costituire un'importante componente del valore se adeguatamente quantificato. Questo richiede accorgimenti che vanno oltre la considerazione erronea di un'aderenza perfetta alla terapia da parte dei pazienti, o un'aderenza pari a quella rilevata durante uno studio registrativo controllato (13), fermo restando che i dati di aderenza reali saranno disponibili solo in fase post-marketing.

\section{Riduzione dell'incertezza}

Questa dimensione del valore vuole premiare i vantaggi derivati dalla "medicina personalizzata" o per esempio da test diagnostici, in associazione a un farmaco, in grado di predire il livello di risposta alla terapia in modo da ottimizzare l'identificazione di una popolazione target riducendo i costi legati al trattamento o agli eventi avversi e migliorando I'aderenza (14). Un altro vantaggio associato alla riduzione dell'incertezza della risposta da parte del paziente potrebbe tradursi in benefici psicologici, anche se questi sono di difficile misurazione. Anche in questo caso, i vantaggi derivanti dalla riduzione dell'incertezza possono essere incorporati, utilizzando metodologie appropriate e validate all'interno delle valutazioni economiche e del calcolo dell'ICER.

\section{Severità della malattia}

Questa componente del valore fa riferimento alla preferenza degli individui o della popolazione generale per guadagni di salute originati in un gruppo di pazienti che ha una prognosi peggiore, rispetto a un altro gruppo di pazienti con un livello di salute migliore. In altre parole, la qualità di vita di un paziente prima del trattamento influenza l'utilità associata al beneficio atteso.

Questa componente del valore richiama il criterio di fine vita adottato per esempio dal National Institute for Health and Care Excellence (NICE) nel Regno Unito (15). Nel Regno Unito questo criterio prevede che, per un trattamento indicato per una piccola popolazione di pazienti con un'aspettativa di vita inferiore a 24 mesi ed evidenza robusta di estensione della sopravvivenza di almeno 3 mesi rispetto al trattamento standard, il vantaggio di sopravvivenza sia pesato con la stessa qualità di vita di un individuo sano nella stessa classe di età. In Italia abbiamo esempi da cui emerge chiaramente come la rimborsabilità, nel caso di patologie gravi, sia concessa anche in presenza di ICER molto elevati o benefici di salute subottimali.

Oltre a quanto previsto nei criteri di determinazione dell'innovatività, non esistono ad oggi definizioni di severità della malattia da utilizzare in maniera sistematica nella determinazione del valore.

\section{Equità}

Questa dimensione coincide con uno dei tre principi fondamentali del nostro SSN (16). II principio di equità prevede che a tutti i cittadini sia garantita parità di accesso in rapporto a uguali bisogni di salute, indipendentemente dalla collocazione geografica, dal reddito, dallo status. Allo stesso tempo questo principio fondamentale ha il fine di superare le diseguaglianze di accesso dei cittadini alle prestazioni sanitarie. Nonostante l'importanza di questa componente del valore, dal punto di vista politico sono stati fatti relativamente pochi tentativi di integrare formalmente il principio di equità nelle valutazioni economiche, che nella versione convenzionale valorizzano principalmente un principio di efficienza rispetto a quello di equità. Alcuni esempi di considerazione di elementi di equità riguardano considerazioni ad hoc rispetto a malattie rare, "bisogni insoddisfatti" o severità della malattia, come discusso in precedenza. Questi concetti rientrano generalmente in quello che si definisce "bisogno insoddisfatto" (unmet need) (17) che, pur con alcune differenze nelle varie accezioni, comprende la disponibilità di trattamenti efficaci, la severità o burden della malattia, l'epidemiologia della popolazione.

Negli Stati Uniti l'Institute for Clinical and Economic Review ha proposto di includere questioni riguardanti l'equità consentendo agli esperti di adeguare le soglie di rapporto costo-efficacia in base a "considerazioni di contesto" (18). Altri autori hanno proposto di quantificare il trade-off (19) tra miglioramento del livello di salute totale e distribuzione di costi ed esiti nei vari gruppi di popolazione oppure di studiare la distribuzione del costo-opportunità tra i vari gruppi della popolazione (20) a seguito dell'adozione di una tecnologia.

Questi aspetti fanno riferimento all'equità da garantire su aree terapeutiche diverse. In Italia, in realtà, quello che preoccupa maggiormente è la (dis)equità di accesso, anche allo stesso farmaco, tra Regioni diverse. La cosiddetta "rimborsabilità debole", come sostengono Armeni e Costa (21), che deriva da un disallineamento temporale e decisionale tra rimborsabilità, finanziamento e diffusione di un nuovo farmaco sul territorio nazionale. Certo è che per ridurre sensibilmente le disuguaglianze nell'accesso legate a questo punto occorrerebbe una revisione profonda della governance farmaceutica nel nostro Paese.

\section{Spillover scientifico}

Questa componente del valore intende premiare l'innovatività scientifica di una molecola che presenta, per esempio, un nuovo meccanismo di azione in grado di rispondere ai bisogni di un sottogruppo di pazienti, di avviare una nuova classe terapeutica o, in generale, quando i progressi scientifici prodotti non si limitano interamente a coloro che li realizzano (22). Esistono ad oggi studi che provano a quantificare le preferenze dei pazienti per una modalità di somministrazione (per es. iniezione sottocutanea) rispetto a un'altra (per es. farmaco orale) in setting sperimentali (23). Altri lavori si sono focalizzati sulla misurazione dei benefici di salute associati 
a un trattamento che travalicano il paziente stesso e riguardano, per esempio, il caregiver, come lo studio di Al-Janabi e colleghi relativo al vaccino anti-meningococco (24). Rispetto all'operazionalizzazione e inserimento di questa componente in una valutazione economica convenzionale, che di fatto trascura l'esternalità sull'innovazione futura, restano comunque ampi margini di studio e ricerca.

\section{Altri elementi}

C'è un ultimo "petalo" nel diagramma proposto da AIFA che riguarda altri elementi probabilmente residuali o che riguardano solo alcune classi di medicinali. Questi elementi sono relativamente nuovi nel dibattito sulla determinazione del valore.

Per esempio, in ambito infettivologico, ridurre l'ansia associata al rischio di diffusione futura di una malattia, sia tra gli esposti che tra i non esposti, può essere importante per la società, anche se il numero atteso di casi è basso. Questo elemento è stato denominato riduzione della "paura di contagio" (25) e può essere quantificato usando delle survey per stabilire la disponibilità a pagare degli individui per eliminare la possibilità di esposizione a un agente infettivo.

Un'altra estensione proposta rispetto a un'analisi di costoefficacia "tradizionale" riguarda quello che è conosciuto in letteratura, non solo farmacoeconomica ma anche finanziaria, come option value (valore dell'opzione). Questo elemento è particolarmente importante in aree patologiche che affrontano fasi di rapida innovazione. Per esempio, in oncologia il panorama delle potenziali alternative terapeutiche si è evoluto di recente grazie all'avvento dell'immunoterapia o delle terapie cellulari (CAR-T, Chimeric Antigen Receptor T-Cell therapy). In questo contesto, un medicinale che consenta ai pazienti di sopravvivere fino alla commercializzazione di una promettente terapia in sperimentazione ha un valore aggiuntivo, il "valore dell'opzione", definito come il vantaggio di traghettare il paziente fino alla prossima innovazione. Esistono pochi esempi ad oggi, nel melanoma metastatico $(26,27)$, carcinoma renale e carcinoma polmonare non a piccole cellule (NSCLC) (28), di studi volti a stimare l'option value, ovvero il beneficio delle terapie al di là del controllo immediato del tumore nel dare ulteriore sopravvivenza ai pazienti che arriveranno così a beneficiare di ulteriori progressi nella ricerca e nella cura della loro patologia.

Anche in questo caso, le conseguenze e implicazioni metodologiche della considerazione dell'option value come dimensione di valore a sé stante, o come variabile da considerare nella quantificazione dell'ICER, rimane un tema di dibattito all'interno della comunità scientifica di riferimento e delle agenzie di HTA.

\section{Conclusione}

Diverse autorità di HTA hanno mostrato interesse per questi nuovi approcci alla definizione del valore e AIFA si inserisce, da questo punto di vista, a pieno titolo in un dibattito vivace e interessante a livello internazionale.

Va chiarito ora, anche con l'aiuto dei ricercatori, quali siano le metodologie e le tecniche più adatte nel nostro contesto per l'identificazione, la valutazione e l'integrazione di questi elementi del valore, soprattutto i più innovativi, durante la fase di assessment di una tecnologia. Più in generale, la considerazione di molteplici dimensioni del valore potrebbe richiedere inoltre la ridefinizione di regole decisionali chiare ed esplicite da applicare nella fase di appraisal, durante il processo di negoziazione di nuovi farmaci da rimborsare all'interno del SSN. Le possibili alternative, in ordine decrescente di fattibilità di implementazione includono:

A) la discussione di questi elementi in un processo deliberativo basato sul dibattito tra i membri esperti delle commissioni;

B) I'utilizzo di un approccio Multi-Criteria Decision Analysis (MCDA), attraverso il quale le diverse dimensioni del valore possano essere identificate, valutate e sintetizzate attraverso una metodologia strutturata ed esplicita (29);

C) I'utilizzo del Net Monetary Benefit (NMB) come singola regola decisionale di riferimento.

Se da una parte la percorribilità di ciascuno di questi approcci nel contesto italiano rimane da verificare, si ritiene che l'apertura a nuove dimensioni del valore richieda contemporaneamente una revisione dell'attuale organizzazione del processo decisionale, in modo da garantire trasparenza e coerenza nelle decisioni tra aree terapeutiche e nel tempo. Ognuna delle strategie menzionate ha pro e contro e il dibattito deve necessariamente svilupparsi e continuare. Rispetto ad alcuni anni fa però, non dobbiamo più discutere del se, ma del come misurare e integrare questi elementi del valore in un quadro multidimensionale di valutazione delle tecnologie sanitarie. E questo rappresenta un grosso passo avanti per tutti.

\section{Ringraziamenti}

Questo testo è frutto delle riflessioni proposte nell'ambito di un corso ECM dal titolo "Farmacisti ed Ematologi Executive Learning Initiative", svoltosi presso SDA Bocconi e organizzato con il supporto incondizionato di Janssen-Cilag Spa.

\section{Disclosures}

Financial support: The authors declare no financial support. Conflict of interest: The authors declare no conflict of interest.

\section{Bibliografia}

1. Kristensen F. EUnetHTA and health policy-making in Europe. Euro-Health. 2006;12:36-8.

2. Methods for the economic evaluation of health care programmes. Michael F. Drummond, Mark J. Sculpher, Karl Claxton, Oxford University Press, United Kingdom 2015 4th Edition.

3. AIFA. Le valutazioni economiche sottomesse ad AIFA nei dossier di richiesta della rimborsabilità e del prezzo ( $P \& R)$. https://www.aifa.gov.it/documents/20142/1028586/valutazioni_ economiche_AIFA_PR_11_2019.pdf/8114ff8c-f4a6-60e2-6c6a7789d6539204

4. Husereau D, Drummond M, Petrou S et al. Consolidated health economic evaluation reporting standards (CHEERS) statement. Cost Eff Resourc Alloc. 2013;11(1):6. 
5. Sullivan SD, Mauskopf JA, Augustovski F et al. Budget impact analysis-principles of good practice: report of the ISPOR 2012 Budget Impact Analysis Good Practice II Task Force. Value Health. 2014;17(1):5-14.

6. Ciani O, Federici CB. Value lies in the eye of the patients: the why, what, and how of patient-reported outcomes measures [published online ahead of print, 2020 Jan 10]. Clin Ther. 2020;42(1):25-33. doi:10.1016/j.clinthera.2019.11.016

7. Neumann PJ, Cohen JT. QALYs in 2018-Advantages and Concerns. JAMA 2018;319(24):2473-4. doi:10.1001/jama.2018. 6072

8. Sculpher MS, Claxton K, Pearson SD. Developing a value framework: the need to reflect the opportunity costs of funding decisions. Value Health. 2017;20(2):234-9.

9. Jommi C, Armeni P, Costa F et al. Implementation of value-based pricing for medicines. Clin Ther. 2020 Jan;42(1):1524.

10. ISPOR. 2019 TOP 10 HEOR TRENDS. https://www.ispor.org/ docs/default-source/about-ispor/ispor-top-10-heor-trends-2019 00120190107_2-2.pdf?sfvrsn=c8d9948b_0

11. Whitehead SJ, Ali S. Health outcomes in economic evaluation: the QALY and utilities. Br Med Bull. 2010;96:5-21.

12. Jan S. Proceduralism and its role in economic evaluation and priority setting in health. Soc Sci Med. 2014 May;108:257-61.

13. Hughes $D$, Cowell W, Koncz $T$ et al. Methods for integrating medication compliance and persistence in pharmacoeconomic evaluations. Value Health. 2007;10:498-509.

14. Goldman DP, Gupta C, Vasudeva E et al. The value of diagnostic testing in personalized medicine. Forum Health Econ Policy. 2013;16:S87-99.

15. National institute for Health and Clinical Excellence. Appraising life-extending, end of life treatments. https://www.nice.org. uk/guidance/gid-tag387/documents/appraising-life-extending-end-of-life-treatments-paper2

16. Ministero della Salute. I principi del Servizio sanitario nazionale (SSN). http://www.salute.gov.it/portale/lea/dettaglioContenutiLea.jsp?lingua=italiano\&id $=5073 \&$ area $=$ Lea\&menu=vuoto

17. Vreman RA, Heikkinen I, Schuurman A et al. Unmet medical need: an introduction to definitions and stakeholder perceptions. Value Health. 2019;22(11):1275-82.

18. ICER, 2020. https://icer-review.org/announcements/icer-seekspublic-input-for-2020-value-assessment-framework/
19. Verguet S, Kim JJ, Jamison DT. Extended cost-effectiveness analysis for health policy assessment: a tutorial. Pharmacoeconomics. 2016;34:913-23.

20. Asaria M, Griffin S, Cookson R. Distributional cost-effectiveness analysis: a tutorial. Med Decis Making. 2016;36:8-19.

21. Armeni P, Costa F. Farmaci e rimborsabilità debole: i legami mancanti per una tutela equa del diritto alle terapie. II Sole 24 ORE Sanità 25 Ottobre 2018. https://www.sanita24.ilsole24ore.com/art/dal-governo/2018-10-25/farmaci-e-rimborsabilita-debole-legami-mancanti-una-tutela-equa-diritto-terapie120907.php?uuid=AEwG1XVG

22. Garrison LP, Kamal-Bahl S, Towse A. Toward a broader concept of value: identifying and defining elements for an expanded cost-effectiveness analysis. Value Health. 2017;20:213-6.

23. Schoenen J, Pascual J, Rasmussen S et al. Patient preference for eletriptan $80 \mathrm{mg}$ versus subcutaneous sumatriptan $6 \mathrm{mg}$ : results of a crossover study in patients who have recently used subcutaneous sumatriptan. Eur J Neurol. 2005 Feb;12(2):10817.

24. Al-Janabi H, Van Exel J, Brouwer $\mathrm{W}$ et al. Measuring health spillovers for economic evaluation: a case study in meningitis. Health Econ. 2016 Dec; 25(12):1529-44.

25. Lakdawalla DN, Doshi JA, Garrison Jr LP et al. Defining elements of value in health care-a health economics approach: an ISPOR Special Task Force report [3]. Value Health. 2018;21(2):131-9.

26. Li M, Basu A, Bennette $C$ et al. How does option value affect the potential cost-effectiveness of a treatment? The case of ipilimumab for metastatic melanoma. Value Health. 2019;22(7): 777-84.

27. Thornton Snider J, Seabury S, Tebeka MG et al. The option value of innovative treatments for metastatic melanoma. Forum Health Econ Policy. 2018 Jun 21;21(1).

28. Thornton Snider J, Batt K, Wu Y et al. The option value of in novative treatments for non-small cell lung cancer and renal cell carcinoma. Am J Manag Care. 2017 Oct 1;23(10):e340e346.

29. Kolasa K,Zah V, KowalczykM. How can multicriteria decisionanalysis support value assessment of pharmaceuticals? - Findings from a systematic literature review. Expert Rev Pharmacoecon Outcomes Res. 2018;18(4):379-91. doi:10.1080/14737167. 2018.1467759 